Open Access

\title{
Perceptions of the causes of eating disorders: a comparison of individuals with and without eating disorders
}

\author{
Elizabeth H. Blodgett Salafia*, Maegan E. Jones, Emily C. Haugen and Mallary K. Schaefer
}

\begin{abstract}
Background: In this study, we examined perceptions regarding the causes of eating disorders, both among those with eating disorders as well as those without. By understanding the differences in perceived causes between the two groups, better educational programs for lay people and those suffering from eating disorders can be developed.

Method: This study used open-ended questions to assess the beliefs of 57 individuals with self-reported eating disorders and 220 without. Participants responded to the questions, "What do you think was (were) the cause(s) of your eating disorder?" and "What do you think is (are) the cause(s) of eating disorders?".

Results: A list of possible codes for the causes of eating disorders was created based on a thorough review of the literature. A manually-generated set of eight codes was then created from individuals' actual responses. Frequencies and chi square analyses demonstrated differences in rates of endorsement between those with eating disorders and those without. Participants with eating disorders most frequently endorsed psychological/emotional and social problems, with genetics/biology and media/culture ideals least endorsed. Participants without eating disorders most frequently endorsed psychological/emotional problems and media/culture ideals, with traumatic life events and sports/health least endorsed. There was a difference between groups in the endorsement of the media as a cause of eating disorders, suggesting that those without eating disorders may overly attribute the media as the main cause while those with eating disorders may not be fully aware of the media's impact. Additionally, while both groups highly endorsed psychological/emotional problems, there was a noticeable stigma about eating disorders among those without eating disorders.
\end{abstract}

Conclusions: There were noteworthy differences between samples; such differences suggest that there is a need for more education on the topic of eating disorders. Furthermore, despite empirical support for the effects of genetics, sports, and family factors, these were infrequently endorsed as causes of eating disorders by both groups. Our results suggest that there is a need for more education regarding the factors associated with eating disorders, in order to reduce the stigma surrounding these disorders and to potentially aid the treatment process.

Keywords: Eating disorders, Perceptions, Causes, Media, Psychological and emotional problems, Stigma, Education

\footnotetext{
* Correspondence: Elizabeth.Salafia@ndsu.edu

Department of Human Development and Family Science, North Dakota State University, 283-H EML Hall, \#2615, P.O. Box 6050, Fargo, North Dakota 58108, USA
} nternational License (http://creativecommons.org/licenses/by/4.0/), which permits unrestricted use, distribution, and reproduction in any medium, provided you give appropriate credit to the original author(s) and the source, provide a link to the Creative Commons license, and indicate if changes were made. The Creative Commons Public Domain Dedication waiver (http://creativecommons.org/publicdomain/zero/1.0/) applies to the data made available in this article, unless otherwise stated. 


\section{Background}

Eating disorders have increasingly become the focus of research studies due to their prevalence, especially in Western cultures. Of the adolescent and young adult populations in the United States, for example, between .3 and $.9 \%$ are diagnosed with anorexia nervosa (AN), between .5 and $5 \%$ with bulimia nervosa $(\mathrm{BN})$, between 1.6 and $3.5 \%$ with binge eating disorder (BED), and about $4.8 \%$ with eating disorder otherwise not specified (EDNOS) [1-4]. According to the fifth edition of the DSM, individuals that do not fit the criteria for AN, BN or BED are diagnosed with sub-threshold or atypical conditions that fit under other specified feeding or eating disorder (OSFED) [5]. Due in part to decreased thresholds for the diagnoses of $\mathrm{AN}, \mathrm{BN}$ or BED in the DSM-V, rates of OSFED have been found to be lower than previous rates of EDNOS, while the rates of AN, $\mathrm{BN}$ or BED have stayed the same or slightly increased [6]. Furthermore, the age at onset is concerning, as most eating disorders originate during adolescence [4]. Despite the potentially serious health consequences that result from disordered eating [7], many in the general public believe that issues with eating are due to personal shortcomings $[8,9]$. This creates a foundation of stigma regarding why individuals develop an eating disorder (e.g., to be "skinny") and the purpose the disorder serves (e.g., to gain control). Such stigma may dishonor the actual experience of those who have lived with an eating disorder, as people could assume eating disorders are self-inflicted. In turn, those developing unhealthy habits may be discouraged from seeking help [10].

Previous research has identified biological, psychological, and sociocultural factors related to the development of eating disorders. However, it is important to explore individual narratives to identify similarities and differences among individuals with and without eating disorders. Obtaining such knowledge can help scholars determine the public's educational needs and better target missing gaps in their knowledge. More accurate information may reduce stigma regarding eating disorders, which may in turn encourage those experiencing symptoms to seek help sooner, as they may no longer fear the negative feedback from peers and family that such stigma causes.

\section{Factors that contribute to eating disorders identified by research}

Research has identified many risk factors, ranging from individual to sociocultural, that contribute to the development of eating disorders. Based on empirical literature, we present some of the most salient factors below.

\section{Individual factors}

Genetics and biology are individual factors that play a role in the development of eating disorders. Genetic contributions to the development of eating disorders have been suggested by twin studies, with heritability estimates ranging from 0.39 to 0.74 , depending on the disorder [11]. Abnormalities in the regulation of certain neurochemicals, such as 5-Hydroxytryptamine (HT) and the serotonin-transporter-linked polymorphic region (5-HTTLPR), have been closely linked with eating disorders [11-13]. Further, recent research has identified mutations on two specific genes that have been associated with increased risk of developing eating disorders in families: estrogen-related receptor $\alpha$ (ESRRA) and histone deacetylase 4 (HDAC4) [14]. In addition, early puberty has also been associated with disordered eating behaviors, potentially due to increases or irregularities in circulating sex hormones, especially estrogen $[15,16]$.

Body dissatisfaction has been commonly identified as an influential risk factor for eating disorders. Individuals dissatisfied with their bodies are at an increased risk of engaging in disordered eating behaviors such as bingeing and purging in order to gain satisfaction and move closer to the thin ideal $[14,17]$. Engaging in dieting behaviors also increases the risk for the occurrence of eating pathology such as binge eating and purging $[15,18]$.

Researchers have recognized perfectionism as a specific risk factor in the development of eating disorders, as this personality trait may lead to a persistent pursuit of the thin ideal $[15,19,20]$. Perfectionism can also be a maintenance factor for disordered eating since it promotes dieting, bingeing, and purging, and enhances eating disorder symptoms, particularly when combined with low self-esteem [12, 15]. Similarly, research has shown that negative affect in general, such as high levels of stress, guilt, hostility, anger, anxiety, and depressed mood, is associated with increases in eating disorder symptoms $[12,13,17-21]$.

Sexual, physical, and emotional abuse have all received empirical support as risk factors for psychiatric difficulties, which can include eating disorders [22]. Specifically, research has shown that sexual abuse can occur in $29 \%$ of individuals with eating disorders, and physical abuse may occur in $57 \%$ of individuals [23, 24]. Additionally, emotional abuse is a significant predictor of eating disorder symptoms among women when other types of abuse are controlled for, suggesting that emotional abuse may be particularly salient $[12,25]$.

\section{Sociocultural factors}

Many sociocultural factors affect the development of eating disorders. In families, for example, mothers' and fathers' own body dissatisfaction and dieting behaviors have been associated with their children's eating-related attitudes and behaviors [26, 27]. Parental weight-related teasing, negative comments about body shape, pressure to lose weight, and encouragement to diet have also 
been associated with body dissatisfaction, dieting, disordered eating behaviors, and eating disorders among both females and males [12, 15, 26, 28-31]. Furthermore, parents who engage in high levels of parental control, expressed emotionality, critical comments, hostility, or emotional overinvolvement and negate their child's emotional needs are more likely to have children who develop eating disorders [12, 32].

Peer influences on the development of eating disorders can also be broken down into a variety of factors. Peer pressure to conform to cultural ideals has been consistently identified as an important factor associated with the development of disordered eating behaviors, especially among adolescents [29,33]. In particular, girls may learn attitudes and behaviors from their peers, such as the importance of being thin and dieting behaviors, through modeling, teasing, and conversations about body image and eating [12, 33]. Similarly, romantic partners play a significant role in the development of eating disorders through negative comments about appearance and encouragement to lose weight, which can lead to weight concerns, body dissatisfaction, and disordered eating behaviors among both men and women [34, 35].

It is also worth mentioning that eating disorders among athletes are common, as there is a large focus not only on being in shape, but on being the fittest and therefore the "best" [36, 37]. There is an even greater risk of developing an eating disorder with participation in certain competitive sports that focus on leanness, such as gymnastics [38]. Athletes who believe that being leaner will increase their performance are more likely to engage in disordered eating [39]. This belief may be encouraged or reinforced by coaches and instructors, further increasing athletes' risk for developing disordered habits [40].

Lastly, the media has an influential, if often controversial, role in the development of eating behaviors due its representation of the thin ideal. There is support that, regardless of the level of internalized thin ideal, women who were warned that a thin media image was altered experienced lower body dissatisfaction in comparison to those who were not warned the image was altered [41]. A preference for a thin and virtually unattainable body has been associated with the development of eating disorders, particularly AN [42].

A relatively small number of studies have examined individuals' perceptions regarding the causes of eating disorders $[10,36,43-55]$. Some studies have solely focused on the perceptions of either the general public [10,43-47] or those with eating disorders [36, 37, 50-55]. Both types of studies have identified a common set of risk factors, with public perceptions and the perceptions of individuals with eating disorders varying slightly [e.g. 48,49]. Overall, both populations have a basic understanding of what eating disorders are and characteristics of each eating disorder
[10, 36, 43-55]. However, despite this knowledge, many adults without eating disorders may be unsympathetic to those suffering from eating disorders, believe that having an eating disorder would not be distressing, and report that eating disorders are not difficult to treat [9].

\section{Public perceptions of factors that contribute to eating disorders}

The studies to date that have focused on identifying public perceptions of the factors associated with the development of eating disorders have surveyed individuals drawn from communities or schools. Typically, these samples have been quite large, numbering over 100 [43, 44] or even several hundred $[10,45,46]$, and have included both females and males [10, 43, 44, 46-48]. Despite the importance of large samples, all of these studies have been limited in that the researchers did not ask open-ended questions; rather, participants responded to forced-answer questions where they either had to identify which item was a cause of eating disorders or identify to what degree a particular item was a cause.

The public commonly places blame on individuals with eating disorders, suggesting that they have control over their "self-inflicted" illnesses [48]. Of the individual factors associated with the development of eating disorders, the majority of people who do not have eating disorders identify psychological explanations such as emotional state, personality, and low self-esteem [10, 43, 46-48]. The general public also believes that individuals' own behaviors and attitudes related to body image such as dieting, a desire to be thin, and body image distortion are important factors in the development of eating disorders [43, 47, 48]. Traumatic events, genetics, and sexual abuse were rarely discussed or, if they were mentioned, rated low on the level of significance in causing eating disorders [10, 47].

Although sociocultural factors are less commonly identified as causal factors of eating disorders among the general public, a few factors have received support. Of all the sociocultural factors, family issues were the factors most often identified [43, 46, 48, 49]. Pressure from friends as well as social isolation and loneliness were also perceived to be factors contributing to eating disorders [46, 47]. In one study, the portrayal of thin women in the media was a highly significant cause endorsed by adult women [45].

\section{Perceptions of individuals with eating disorders regarding causes}

In contrast to studies investigating the perceptions of the general public regarding factors associated with the development of eating disorders, most studies we found that focused on individuals with eating disorders used open-ended measures, either via interview or questionnaires. Despite this, one pitfall of the research to date is that it has often involved relatively small sample sizes, ranging from 15 to 36 
[36, 37, 49-51]. Only two studies have included samples over 50 individuals $[52,53]$. Additionally, almost all of these studies have focused exclusively on women, with only two including a limited number of men [37, 50]. Furthermore, although research has included assessments of individuals with AN $[36,50,54]$ and BN $[53,55]$ or both $[49,51,52]$, studies have failed to examine if differences existed in the perceptions of those with $\mathrm{AN}$ versus $\mathrm{BN}$, or include individuals with other eating disorders such as BED, EDNOS, or OSFED.

Similar to public perceptions of causal factors, people with eating disorders also identify individual and sociocultural factors. Individual factors commonly identified among samples of those who were diagnosed with eating disorders include perfectionism, emotional problems or distress, stress, unhappiness with appearance, high expectations of self, and lack of control [36, 48, 50-54]. Behaviors and attitudes related to body image, such as weight loss activities, body image distortion, and a belief that thinness equals happiness, were also frequently identified as factors that related to the development of their disorders $[48,53,55]$. Hereditary factors and sexual abuse were not indicated.

Sociocultural influences identified by individuals with eating disorders included the media, family, peers, and sports. Although rarely mentioned, the media was occasionally identified as playing a role through the importance it places on thinness and self-comparison to the thin ideal $[36,37]$. Family factors, in contrast, were often cited and included poor parental care, controlling parents, poor relationship with parents, family tension or high amounts of conflict, critical family environment, emotional abuse, and an emphasis on weight [36, 37, 48-51, 53, 55]. Factors associated with peers and sports were also common and included receiving comments or pressure from friends and coaches about appearance, a need to lose weight for sports performance, and poor relationships with peers $[36,37,53,55]$.

\section{Comparisons of individuals with and without eating disorders}

We could only find two studies that examined the perceptions of both individuals with and without eating disorders. First, Haworth-Hoeppner [49] interviewed 21 women with an eating disorder (either AN or BN) and 11 without, asking open-ended questions about the development of eating disorders. In this study, no comparisons were made across the two groups, likely due to the qualitative nature of the project as well as the small sample size. Second, Holliday and colleagues [48] used larger samples of individuals with and without $\mathrm{AN}$ and made comparisons across groups regarding the causes of eating disorders and the most important causes. However, this study was limited in that it did not allow participants to describe their own beliefs.
Instead, participants responded to a list of eighteen preidentified causes of eating disorders, which did not allow for individual perspectives and greater depth into the complexity of eating disorders.

\section{The present study}

With the prevalence of eating disorders and young age of onset, examining people's perceptions of the factors contributing to eating disorders is important. Such efforts can enhance public education and potentially decrease the stigma surrounding eating disorders. The present study specifically examined the differences between what people with and without eating disorders perceived to be the causes of eating disorders in order to better understand people's experiences with eating disorders as well as to better educate the larger population. We also examined differences regarding the causes of eating disorders according to type of eating disorder, including $\mathrm{AN}, \mathrm{BN}$, both, and other (e.g., BED, EDNOS, or OSFED). This study strengthens existing research by utilizing qualitative, open-ended responses as opposed to forced-answer questionnaires so that participants could identify causes using their own opinions.

\section{Method}

\section{Participants and procedure}

This study was reviewed and approved by the university's Institutional Review Board. Our sample was recruited from flyers and emails distributed at local universities as well as from flyers distributed to local hospitals and clinics in a medium-sized, Midwestern U.S. city. A secure Internet link was provided, which participants used to indicate consent, provide demographic information, and answer several open-ended questions. All participants were first asked, "Do/did you have an eating disorder?" with the answer choices of "yes, currently," "yes, in the past," and "no." Individuals who answered as having an eating disorder, whether past or current, were asked to specify which eating disorder they had/have and for how long.

The total sample consisted of 277 participants: 57 individuals who had a past or current eating disorder and 220 who did not. Consistent with the ethnic composition of the city, most of the sample identified themselves as White (93\%). There were 234 females $(84.5 \%)$ and 43 males $(15.5 \%)$. The age range of participants was from 18 to 51 $(\mathrm{M}=22.39, \mathrm{SD}=5.77)$.

\section{Sample with eating disorders}

Of the 57 individuals who had an eating disorder, 26 had AN (46\%), 12 had BN (21\%), 11 had both AN and BN (19\%), and 8 had another type of eating disorder such as BED or EDNOS/OSFED (14 \%). Participants reporting having an eating disorder from between 4 months and 22 years $(M=3.70$ years, $S D=4.55$ years). Similar to the 
demographics of the entire sample, $93 \%$ identified as White, and the majority of individuals in this sample were female $(96.5 \% ; n=55)$. Participants ranged in age from 18 to $47(\mathrm{M}=23.70, \mathrm{SD}=5.84)$.

\section{Sample without eating disorders}

Of the 220 individuals who did not have an eating disorder, $93 \%$ identified as White. In addition, $81 \%$ identified as female $(n=179)$. Participants ranged in age from 18 to 51 $(M=22.05, S D=5.71)$. In terms of ethnicity and age, both samples were similar; there were no statistically significant differences between samples $(p=.80$ and $p=.11$, respectively). There was, however, a statistically significant difference in gender $(p=.01)$.

\section{Survey questions and compensation}

After completing a series of demographic questions using the secure Internet link, individuals who had an eating disorder were asked the open-ended question, "What do you think was (were) the cause(s) of your eating disorder?" Individuals who did not have an eating disorder were asked a similar open-ended question, "What do you think is (are) the cause(s) of eating disorders?" These participants were then asked to report why they believed that these were the causes or how they learned about them. All participants were invited to participate in a random drawing for one of four $\$ 50$ giftcards. Interested individuals were given another secure Internet link to provide their contact information if they wished to enter the drawing; this was done to keep the survey responses anonymous.

\section{Results}

\section{Coding of participants' reponses}

We initially created a list of possible codes for the causes of eating disorders commonly specified in previous research articles (as identified by overview articles on the risk factors or causes of eating disorders [e.g., 12, 15]). This provided us with a basic framework for content analysis [56]. Next, we manually generated a set of codes from actually reading individuals' responses to the questions, "What do you think was (were) the cause(s) of your eating disorder?" and "What do you think is (are) the cause(s) of eating disorders?" Thus, we were able to identify a unique but relevant set of eight key themes. The eight themes that emerged from the data were: 1) traumatic life events, 2) family problems, 3) social problems, 4) psychological and emotional problems, 5) genetics and biology, 6) media and culture ideals, 7) sports and health, and 8) body image and eating.

Participants' responses were then grouped under each of these categories. Many participants identified multiple causes of eating disorders, which were therefore grouped under multiple categories. The responses were coded independently by three research assistants, then checked by an additional research assistant and the first author for consistency. This was done to ensure interrater reliability [56]. When a difference in coding existed, the research team discussed the differences and mutually agreed upon a solution. See Table 1 for sample responses in each category.

\section{Frequencies of individuals reporting each cause Sample with eating disorders}

A Chi square test for goodness of fit indicated that the participants in this sample showed significantly different rates of endorsement among the causes of eating disorders, $\chi^{2}(7, n=108)=41.63, p<.05$. Specifically, psychological and emotional $(n=30)$ and social problems $(n=22)$ were most frequently endorsed, with the lowest number of endorsements for genetics and biology $(n=2)$ and media and culture ideals $(n=5)$.

Individuals with AN most commonly indicated psychological and emotional problems as the cause $(n=13)$, followed by body image and eating problems $(n=9)$. Individuals with $\mathrm{BN}$ reported psychological and emotional $(n=8)$ and social $(n=7)$ as the primary causes. Those with both $\mathrm{AN}$ and $\mathrm{BN}$ listed all types of problems as causes, so there was not a clear primary cause, although social $(n=5)$ and psychological and emotional problems $(n=4)$ were slightly more frequently endorsed. Finally, those with other eating disorders most frequently cited psychological and emotional problems $(n=5)$ and traumatic life events $(n=3)$. See Table 2 for a complete

Table 1 Specific examples of cited causes of eating disorders

\begin{tabular}{ll}
\hline Coded Category & Causes \\
\hline Traumatic life events & Sexual assault, college entry, abuse \\
Family problems & Comments from family, pressure from parents, need for praise, conversations about weight \\
Social problems & Bad romantic relationship or break-up, pressure from peers, teasing, social isolation \\
Psychological and emotional problems & Stress, depression, anxiety, need for control, perfectionism, low self-esteem \\
Genetics and biology & History of eating disorders in family, chemical imbalance in the brain \\
Media and culture ideals & Thin ideal images and messages \\
Sports and health & Gymnastics or dance, health or exercise class, desire to be healthy, lack of knowledge about nutrition \\
Body image and eating & Drive for thinness, unhappiness with appearance, feeling overweight or unattractive, distorted image \\
\hline
\end{tabular}


Table 2 Frequencies of individuals citing each category for the causes of eating disorders

\begin{tabular}{|c|c|c|c|c|c|}
\hline \multirow[t]{2}{*}{ Category } & \multicolumn{5}{|c|}{ Frequency according to Eating Disorder } \\
\hline & Anorexia & Bulimia & Both & Other & No ED \\
\hline Traumatic life events & $5(19 \%)$ & $3(25 \%)$ & $2(18 \%)$ & $3(38 \%)^{2}$ & $5(2 \%)$ \\
\hline Family problems & $7(27 \%)$ & $2(17 \%)$ & $1(9 \%)$ & 1 (13\%) & $28(13 \%)$ \\
\hline Social problems & $8(31 \%)$ & $7(58 \%)^{2}$ & $5(45 \%)^{1}$ & $2(25 \%)$ & $57(26 \%)$ \\
\hline $\begin{array}{l}\text { Psychological and } \\
\text { emotional problems }\end{array}$ & $13(50 \%)^{1}$ & $8(67 \%)^{1}$ & $4(36 \%)^{2}$ & $5(63 \%)^{1}$ & $141(64 \%)^{1}$ \\
\hline Genetics and biology & $0(0 \%)$ & $0(0 \%)$ & $2(18 \%)$ & $0(0 \%)$ & $18(8 \%)$ \\
\hline Media and culture ideals & $2(8 \%)$ & $0(0 \%)$ & $3(27 \%)$ & $0(0 \%)$ & $104(47 \%)^{2}$ \\
\hline Sports and health & $6(23 \%)$ & $2(17 \%)$ & $2(18 \%)$ & 1 (13\%) & $4(2 \%)$ \\
\hline Body image and eating & $9(35 \%)^{2}$ & $1(8 \%)$ & $3(27 \%)$ & $1(13 \%)$ & $57(26 \%)$ \\
\hline Total sample size & 26 & 12 & 11 & 8 & 220 \\
\hline $\begin{array}{l}\text { Total number of } \\
\text { responses given }\end{array}$ & 50 & 23 & 22 & 13 & 414 \\
\hline
\end{tabular}

Notes. Numbers in the table represent how many times each cause was cited by each separate sample. Numbers in parentheses in the table represent the proportion of individuals within each sample reporting that particular cause. Total sample size refers to how many individuals were in each sample. Total number of responses given refers to how many different answers were provided by each sample (i.e., some individuals provided multiple causes of eating disorders).

1,2 indicate two most frequently endorsed causes for each eating disorder type

listing of the frequencies of individuals citing each causal category.

\section{Sample without eating disorders}

A Chi square test for goodness of fit indicated that the participants in this sample showed significantly different rates of endorsement among the causes of eating disorders, $x^{2}(7, n=414)=326.95, p<.05$. Specifically, psychological and emotional problems $(n=141)$ and media and culture ideals $(n=104)$ were most frequently endorsed, with the lowest number of endorsements for family problems $(n=28)$, genetics and biology $(n=18)$, traumatic life events $(n=5)$, and sports and health $(n=4)$. Clearly, this sample differed from the sample of individuals with eating disorders in what they viewed as the primary causes. See Table 2 for the frequencies.

\section{Differences between samples}

Chi square tests for independence indicated that there was not a significant relationship between type of eating disorder (AN, BN, both, or other) and the causes specified. Furthermore, there were no significant relationships among each pairing of eating disorder sub-groups. The lack of statistically significant findings here could be the result of our small sample sizes for each group. See Table 3 for a summary of results from these chi square tests for independence.

Of particular noteworthiness, results from a chi square test of independence indicated that there was a significant relationship between eating disorder versus non-eating disorder groups and the causes specified, $\chi^{2}(7, n=522)=$ $77.96, p<.05$, Phi $=.39$. This suggests that individuals with and without eating disorders had significantly different views regarding the causes of eating disorders, with each group likely to endorse causes at different rates. In conducting follow-up analyses of each cause separately, we found significant differences in the endorsement of family problems $\left(X^{2}(1, n=39)=7.41, p<.05\right)$, social problems $\left(\chi^{2}(1, n=79)=15.51, p<.05\right)$, psychological and emotional problems $\left(\chi^{2}(1, n=171)=72.05, p<.05\right)$, genetics and biology $\left(\mathrm{X}^{2}(1, n=20)=12.80, p<.05\right)$, media and culture $\left(\chi^{2}(1, n=109)=89.92, p<.05\right)$, and body image and eating $\left(X^{2}(1, n=71)=26.04, p<.05\right)$ among those with and without eating disorders. More specifically, individuals with eating disorders more often endorsed family problems, and social problems while individuals without eating disorders more often endorsed psychological and emotional problems, genetics and biology, media and culture, and body image and eating.

Additionally, there were significant relations between each individual type of eating disorder versus non-eating disorder and the causes specified. See Table 3 for these results. This suggests, for example, that individuals without eating disorders had different levels of endorsement for each cause than the group of individuals with AN. The same was true for the sub-groups of $\mathrm{BN}$, both, and other, when compared to individuals without eating disorders.

\section{Discussion}

This is the only known study that assessed subjective perceptions of the causes of eating disorders among a relatively large sample of individuals with and without eating disorders. The results support differences between the general public and individuals suffering from eating disorders, which hopefully can be used to provide proper education. Specifically, the general public largely believed that the 
Table 3 Chi square tests of independence for the relationships between type of eating disorder or no eating disorder and causes specified

\begin{tabular}{lll}
\hline Model & $X^{2}(\mathrm{~d})$ & $\Phi$ \\
\hline $\begin{array}{l}\text { Differences between Samples with } \\
\text { Eating Disorders and without Eating }\end{array}$ & $77.96^{*}(7)$ & .39 \\
Disorders on the Causes Specified & & \\
(with each eating disorder separated) & $98.39^{*}(28)$ & .43 \\
AN vs. No ED & $56.78^{*}(7)$ & .35 \\
BN vs. No ED & $38.64^{*}(7)$ & .30 \\
Both vs. No ED & $23.17^{*}(7)$ & .23 \\
Other vs. No ED & $41.33^{*}(7)$ & .31 \\
Differences among Samples with & $23.04(21)$ & .34 \\
Varying Types of Eating Disorders & & \\
on the Causes Specified & & .28 \\
AN vs. BN & $5.73(6)$ & .26 \\
AN vs. Both & $8.90(7)$ & .24 \\
AN vs. Other & $3.66(6)$ & .43 \\
BN vs. Both & $8.18(7)$ \\
BN vs. Other & $1.47(5)$ & .41 \\
Both vs. Other & $6.01(7)$ &
\end{tabular}

Note. ${ }^{*} p<.05$

media causes eating disorders, a perception that is not shared among individuals with an eating disorder. Similarly, sports, body image, and traumatic events were listed less frequently by participants without eating disorders than participants with eating disorders. However, psychological and emotional problems were highly endorsed by all. Together, these findings indicate differences in opinion regarding the causes of eating disorders between those who have an eating disorder and those who do not.

The open-ended questions used in the present study enabled us to gain insight into individuals' personal opinions regarding factors associated with the development of their disorders, ultimately providing a greater understanding for both clinicians and lay people. Psychological and emotional problems were the most frequently reported causes for those with an eating disorder, supporting the need for greater availability of support systems. In considering the perspectives of individuals who had an eating disorder, it is difficult to know if their perceptions align accurately with the actual causes. However, professionals working with these individuals could help assess the discrepancy between perceived and actual causes. For many postmodern therapists, understanding the perception of the eating disorder from the client perspective and helping him or her make meaning of the experience is more important than determining the actual cause of the disorder $[57,58]$. This, therefore, provides reinforcement for the role of psychologists and family therapists within the field of eating disorders, yet many currently lack sufficient training to address eating disorders and instead must refer clients to specialists, who are often expensive and not widely located.

\section{The role of the media}

Our findings revealed a definite contrast between how people with and without eating disorders perceive media as a risk factor for developing an eating disorder. A large percentage of people without eating disorders identified media as a cause ( $47 \%$ ), but only five total participants with eating disorders did. There is a clear separation in the experience of those with eating disorders and with society's conceptualization of them [36, 37]. Thus, it seems that lay individuals may overemphasize the role of the media as one of the main causes of eating disorders, while those with eating disorders may not be fully aware of the potential impact of the media [50]. Whereas specific media variables such as depiction of the thin ideal and unrealistic body standards may be correlated with eating disorders [42], they do not fully explain disordered behaviors. Our findings should be used to educate consumers of media on the complexity of eating disorders, and as evidence for the need to change the types of messages regarding body image ideals that are currently available in the media.

\section{Psychological and emotional problems}

Psychological and emotional problems were one of the highest named causes of eating disorders by both groups, which is consistent with prior research [43, 46, 48]. However, upon close examination of the data, we noticed a contrast between the written answers of those who had eating disorders and those who did not. More specifically, individuals with eating disorders listed personal reasons, such as "a bad relationship that caused a lot of low self-esteem," or simple statements such as "stress, depression." In contrast, there was a negative stigma surrounding some of the answers from participants without eating disorders. These answers included phrases such as "no self-confidence" and "mental disabilities." This difference is worth noting, because it demonstrates a stigma towards those with eating disorders, which may result in a fear of judgment from others that often prevents those suffering from eating disorders to seek help [59]. Reduction of this stigma through educational programs could encourage individuals who are developing disordered eating habits to speak up, as well as encourage friends and family to begin a non-judgmental, supportive dialogue with individuals about their habits.

\section{Other factors}

Traumatic life events were only listed by $2 \%$ of the noneating disorder group, versus $23 \%$ of those with eating disorders. This once again emphasizes the need for education geared towards the general public. However, there is also a 
need for better education for those with eating disorders, as the number of people listing traumatic events was quite low. Many individuals may not make the connection between a traumatic event, such as sexual assault, and the beginning of their disorder, despite empirical support for the effects of abuse [22].

Similar to previous studies, genetics as a cause of disordered eating was only listed by two participants with eating disorders and eight participants without eating disorders $[10,47]$, making it the least endorsed cause. This indicates a need for the dissemination of information regarding the genetic component of eating disorders, as this could potentially help with the negative stigma surrounding eating disorders [60].

Similarly, and in line with previous studies, only twelve participants with eating disorders and 28 participants without listed family problems as a cause of disordered eating $[43,46,48,49]$. There are numerous studies, however, that show the impact that mothers, fathers, and siblings can have on the development of disordered eating in an individual (e.g., [26, 27]). If education efforts could help improve understanding of how eating disorders develop within families, parents and siblings can take steps towards preventing the occurrence of these issues and can work towards developing healthier habits for themselves as well.

Sports and health were also listed more frequently as causes by those with eating disorders (19\%), whereas only $2 \%$ of those without eating disorders mentioned them. However, these numbers are both still low. The general public, and specifically coaches, need to be aware of how an intense focus on the body can lead to negative outcomes and strive to support healthy methods of getting and staying in shape.

Body image was listed as a cause of eating disorders by $26 \%$ of participants without an eating disorder, and $25 \%$ of those with experience with disorder eating; these numbers represent a substantial portion of participants. Poor body image often provides a foundation for the development of an eating disorder $[15,17]$, and understanding what issues underlie an eating disorder can help not only those struggling to recover, but those trying to assist them.

Another highly-endorsed cause of eating disorders was social problems, as $26 \%$ of those without eating disorders and $39 \%$ of those with eating disorders listed them. While these numbers are considerably higher than other groups, only one fourth of those without eating disorders acknowledged social problems as a cause, while a much larger number of those with eating disorders indicated social problems as a cause. However, many individuals may not realize the effect that external events can have on their internal belief systems, once again indicating the need to incorporate this finding into general education, as well as into the treatment process as a way of lessening the blame that those suffering may place on themselves.

\section{Summary of findings}

This study provides insight into the educational resources needed to inform the lay audience regarding eating disorders as well as some factors to consider in the education or prevention of eating disorders among those affected. There is a clear difference between perceived causes of eating disorders from those who have experienced them and those who have not. Those who had not struggled with an eating disorder were more likely to believe that media and cultural ideals influenced eating disorders. For those who had lived with an eating disorder, this was one of the least likely perceived causes. Social problems, in contrast, were frequently listed by participants with eating disorders and less frequently listed by participants without. Genetics and traumatic events were listed most infrequently by both groups, and there were also relatively low levels of endorsement for traumatic life events, sports and health, and family problems among both groups. Both groups listed body image as a fairly frequent cause, and although both groups highly endorsed psychological and emotional problems as causes, there was a clear negative stigma surrounding psychological and emotional problems when listed by non-disordered participants. Improved educational programs should seek to give those who are uninformed a greater understanding of how psychological, social, and relational factors influence those with eating disorders. Increased opportunities for those who have lived with eating disorders to share their stories and perspectives are also needed. With the opportunity to provide first-hand knowledge, these individuals can be an excellent asset for researchers, professionals, and lay people.

\section{Limitations}

Our sample was a relatively homogenous group in terms of gender and ethnicity, so separate analyses could not be conducted examining differences among men and women or among various ethnic groups. Thus, care should be taken when generalizing the results to males and nonwhite individuals. Furthermore, in order to utilize openended questions, no measurement scales were used to determine eating disorder pathology. Therefore, eating disorder status was determined solely by self-report and may not be clinically accurate. In retrospect, it may have been useful to at least provide participants with a self-report survey to assess their eating disorder symptomatology. However, we do note that our sample was recruited not only from local universities but directly from hospitals and clinics that included eating disorder treatment facilities. As a result, we hope that participants were able to appropriately reflect on the nature of their symptomatology. Further, our type of questioning allowed for only two groups of samples, those with eating disorders and those without; individuals who have subclinical symptoms or undiagnosed eating disorders may have been inaccurately placed in the 
category of non-eating disorder due to their own assessment. Similarly, those who identified themselves as having an eating disorder may have been self-diagnosed, and therefore may not technically meet clinical standards for a disorder.

Additionally, two different questions were asked of participants. Specifically, we asked participants with an eating disorder: "What do you think was (were) the cause(s) of your eating disorder?", and we asked participants without an eating disorder: "What do you think is (are) the cause(s) of eating disorders?" This allows individuals to add a personal dimension to their analysis of the causes of eating disorders. As such, they may believe that the cause of their disorder is very different than the cause of someone else's disorder. Similarly, individuals with an eating disorder may have focused more on life events or recent triggers without a reflection on more general risk factors.

Lastly, because this study was completed online, it could be considered relatively impersonal, whereas in-person interviews would have most likely been more in depth. However, because the main interest of the study was to examine participants' instinctive reactions to eating disorders, the completely anonymous online survey was the most beneficial means of execution.

\section{Conclusions}

Despite limitations, this study contributes to the field in a variety of ways. The sample size of those with eating disorders $(n=57)$ is somewhat larger than samples currently in the literature. Furthermore, while many studies focus only on $\mathrm{AN}$ or BN, this study included those with self-reported $\mathrm{AN}, \mathrm{BN}, \mathrm{BED}$ and EDNOS/OSFED, allowing for more inclusive results. It also allowed us to separately assess perceived causes of eating disorders according to the type of eating disorder. For example, individuals with AN most frequently indicated psychological and emotional problems as well as body image and eating problems; individuals with $\mathrm{BN}$ often reported psychological and emotional problems as well as social problems; individuals with both $\mathrm{AN}$ and BN listed all types of problems; and individuals with BED, EDNOS, or OSFED primarily cited psychological and emotional problems as well as traumatic life events. Although these differences in perceptions were not statistically significant, it may suggest that each type of disorder is unique, with potentially unique causes attributed to the disorder. Future research should continue to examine these differences, and education should focus on the unique nature of each type of eating disorder.

The use of an open-ended qualitative assessment allowed for a complete picture of individuals' perceptions of the causes of eating disorders. It also allowed individuals to write about more than one perceived cause of the disorders, which is not always possible with close-ended questions with limited answer options. An additional strength of this study is that it contributes to the relatively small pool of current literature discussing perceptions of eating disorders. Within this limited research, most examine perceptions of the general public or perceptions of those with eating disorders separately. Our study is also one of very few studies to examine differences between these two groups.

Overall, it appears that all individuals would benefit from learning more about eating disorders and their causes. Knowing this could be particularly helpful for individuals going through eating disorder treatment, especially for therapists to use when educating those close to someone struggling with an eating disorder. This could help facilitate greater support and connection between family members and friends, and help to end the stigma surrounding these problems and allow those in trouble to seek help.

\section{Competing interests}

The authors declare that they have no competing interests.

\section{Authors' contributions}

EHBS conceived of and designed the study, developed codes for and analysed the data, and wrote the manuscript. MEJ co-wrote and formatted the manuscript. ECH coded data and co-wrote the manuscript. MKS coded data and co-wrote the manuscript. All authors read and approved the final manuscript.

\section{Acknowledgements}

We thank our undergraduate and graduate research assistants at the North Dakota State University Eating Disorders and Body Image Lab for their assistance with coding the data.

Received: 13 April 2015 Accepted: 4 September 2015

Published online: 15 September 2015

\section{References}

1. Le Grange D, Swanson SA, Crow SJ, Merikangas KR. Eating disorder not otherwise specified presentation in the US population. Int J Eat Disord. 2012:45:711-8

2. Hudson J, Hiripi E, Pope H, Kessler R. The prevalence and correlates of eating disorders in the National Comorbidity Survey Replication. Biol Psychiatry. 2007:61:348-58.

3. Rosen DS. Identification and management of eating disorders in children and adolescents. Pediatr. 2010;126:1240-53.

4. Swanson SA, Crow SJ, LeGrange D, Swendsen J, Merikangas KR. Prevalence and correlates of eating disorders in adolescents: results from the National Comorbidity Survey Replication Adolescent Supplement. Arch Gen Psych. 2011;68:714-23

5. American Psychiatric Association. Diagnostic and statistical manual of mental disorders. 5th ed. Arlington, VA: American Psychiatric Association; 2013.

6. Quick V, Berg KC, Bucchianeri MM, Byrd-Bredbenner C. Identification of eating disorder pathology in college students: a comparison of DSM-IV-TR and DSM-5 diagnostic criteria. Adv Eat Disord. 2014:2:112-24.

7. Arcelus J, Mitchell AJ, Wales J, Nielsen S. Mortality rates in patients with anorexia nervosa and other eating disorders: a meta-analysis of 36 studies. Arch Gen Psych. 2011;68:724-31.

8. Lee NM, Lucke J, Hall WD, Meurk C, Boyle FM, Carter A. Public views on food addiction and obesity: implications for policy and treatment. PLoS One. 2013;8, e74836.

9. Mond JM, Arrighi A. Gender differences in perceptions of the severity and prevalence of eating disorders. Early Int Psych. 2011;5:41-9.

10. Mond JM, Hay PJ. Public perceptions of binge eating and its treatment. Int J Eat Disord. 2008;41:419-26.

11. Yilmaz Z, Hardaway AJ, Bulik CM. Genetics and epigenetics of eating disorders. Adv Genomics Genet. 2015;5:131-50.

12. Polivy J, Herman CP. Causes of eating disorders. Annu Rev Psychol. 2002;53:187-214. 
13. Lee $Y$, Lin PY. Association between serotonin transporter gene polymorphism and eating disorders: a meta-analytic study. Int J Eat Disord. 2010;43:498-504.

14. Cui H, Moore J, Ashimi SS, Mason BL, Drawbridge JN, Han S, et al. J Clin Invest. 2013;123:4706-13.

15. Stice E. Risk and maintenance factors for eating pathology: a meta-analytic review. Psychol Bull. 2002;128:825-48.

16. Zehr JL, Culbert KM, Sisk CL, Klump KL. An association of early puberty with disordered eating and anxiety in a population of undergraduate women and men. Horm Behav. 2007;52:427-35.

17. Wertheim EH, Koerner J, Paxton SJ. Longitudinal predictors of restrictive eating and bulimic tendencies in three different age groups of adolescent girls. J Youth Adolesc. 2001;30:69-82.

18. Leon GR, Fulkerson JA, Perry CL, Keel PK, Klump KL. Three to four year prospective evaluation of personality and behavioral risk factors for later disordered eating in adolescent girls and boys. J Youth Adolesc. 1999;28:181-96.

19. Fairburn CG, Cooper Z, Doll HA, Welch SL. Risk factors for anorexia nervosa: three integrated case-control comparisons. Arch Gen Psychiatry. 1999;56:468-76.

20. Tyrka AR, Waldron I, Graber JA, Brooks-Gunn J. Prospective predictors of the onset of anorexic and bulimic syndromes. Int J Eat Disord. 2002;32:282-90.

21. Tylka TL. The relation between body dissatisfaction and eating disorder symptomatology: an analysis of moderating variables. J Couns Psychol. 2004;51:178-91.

22. Neumark-Sztainer D, Story M, Hannan PJ, Beuhring T, Resnick MD. Disordered eating among adolescents: associations with sexual/physical abuse and other familial/psychosocial factors. Int J Eat Disord. 2000:28:249-58.

23. Treuer T, Koperdák M, Rózsa S, Füredi J. The impact of physical and sexual abuse on body image in eating disorders. Eur Eat Disord Rev. 2005;13:106-11.

24. Wonderlich SA, Rosenfeldt S, Crosby RD, Mitchell JE, Engel SG, Smyth J, et al. The effects of childhood trauma on daily mood lability and comorbid psychopathology in bulimia nervosa. J Traum Stress. 2007;20:77-87.

25. Fischer S, Stojek M, Hartzell E. Effects of multiple forms of childhood abuse and adult sexual assault on current eating disorder symptoms. Eat Behav. 2010;11:190-2.

26. Fulkerson JA, McGuire MT, Neumark-Sztainer D, Story M, French SA, Perny CL. Weight-related attitudes and behaviors of adolescent boys and girls who are encouraged to diet by their mothers. Int J Obes. 2002;26:1579-87.

27. Keery H, Eisenberg M, Boutelle K, Neumark-Sztainer D, Story M. Relationships between maternal and adolescent weight-related behaviors and concerns: the role of perception. J Psychosom Res. 2006;61:105-11.

28. Tremblay L, Lariviere $M$. The influence of puberty onset, body mass index, and pressure to be thin on disordered eating behaviors in children and adolescents. Eat Behav. 2009;10:75-83

29. Wertheim EH, Paxton SJ, Schutz HK, Muir SL. Why do adolescent girls watch their weight? An interview study examining sociocultural pressures to be thin. J Psychosom Res. 1997;42:345-55.

30. Ata RN, Ludden AB, Lally MM. The effects of gender and family, friend, and media influences on eating behaviors and body image during adolescence. J Youth Adolesc. 2007;36:1024-37.

31. Vincent MA, McCabe MP. Gender differences among adolescents in family, and peer influences on body dissatisfaction, weight loss, and binge eating behaviors. J Youth Adolesc. 2000;29:205-21.

32. Kluck AS. Family factors in the development of disordered eating: integrating dynamic and behavioral explanations. Eat Behav. 2008:9:471-83.

33. van den Berg P, Thompson JK, Obremski-Brandon K, Coovert M. The tripartite influence model of body image and eating disturbance: a covariance structure modeling investigation testing the meditational role of appearance comparison. J Psychosom Res. 2002;53:1007-20.

34. Sheets $V$, Ajmere K. Are romantic partners a source of college students weight concern? Eat Behav. 2005;6:1-9.

35. Weller JE, Dziegielewski SF. The relationship between romantic partner support styles and body image disturbance. J Hum Behav Soc Environ. 2004;10:71-92

36. Dignon A, Beardsmore A, Spain S, Kuan A. 'Why I won't eat': patient testimony from 15 anorexics concerning the causes of their disorder. J Health Psychol. 2006;11:942-56.

37. Woods S. Untreated recovery from eating disorders. Adolescence. 2004;39:361-71.
38. Kong $P$, Harris LM. The sporting body: body image and eating disorder symptomatology among female athletes from leanness focused and nonleanness focused sports. J Psychol. 2015;149:141-60.

39. Krentz EM, Warschburger P. A longitudinal investigation of sports-related risk factors for disordered eating in aesthetic sports. Scand J Med Sci Sports. 2013;23:303-10.

40. Thompson RA, Sherman R. Reflections on athletes and eating disorders. Psychol Sport Exerc. 2014;15:729-34

41. Slater A, Tiggemann M, Firth B, Hawkins K. Reality check: an experimental investigation of the addition of warning labels to fashion magazine images on women's mood and body dissatisfaction. J Soc Clin Psychol. 2012;31:105-22.

42. Ahern AL, Bennett KM, Hetherington MM. Internalization of the ultra-thin ideal: positive implicit associations with underweight fashion models are associated with drive for thinness in young women. Eat Disord. 2008;16:294-307.

43. Furnham A, Hume-Wright A. Lay theories of anorexia nervosa. J Clin Psychol. 1992;48:20-36.

44. Furnham A, Manning R. Young people's theories of anorexia nervosa and obesity. Couns Psychol Q. 1997;10:389-414.

45. Mond JM, Hay PJ, Rodgers B, Owen C, Beumont PJ. Beliefs of women concerning causes and risk factors for bulimia nervosa. Aust N Z J Psychiatry. 2004;38:463-9.

46. Smith MC, Pruitt JA, Mann LM, Thelen MH. Attitudes and knowledge regarding bulimia and anorexia nervosa. Int J Eat Disord. 1986;5:545-53.

47. Lee S. How lay is lay? Chinese students' perceptions of anorexia nervosa in Hong Kong. Soc Sci Med. 1997;44:491-502.

48. Holliday J, Wall E, Treasure J, Weinman J. Perceptions of illness in individuals with anorexia nervosa: a comparison with lay men and women. Int J Eat Disord. 2005:37:50-6.

49. Haworth-Hoeppner S. The critical shapes of body image: the role of culture and family in the production of eating disorders. J Marriage Fam. 2000;62:212-27.

50. Button EJ, Warren RL. Living with anorexia nervosa: the experience of a cohort of sufferers from anorexia nervosa 7.5 years after initial presentation to a specialized eating disorders service. Eur Eat Disord Rev. 2001;9:74-96.

51. Patching J, Lawler J. Understanding women's experiences of developing an eating disorder and recovering: a life-history approach. Nurs Inq 2009;16:10-21.

52. Tozzi F, Sullivan PF, Fear JL, McKenzie J, Bulik CM. Causes and recovery in anorexia nervosa: the patient's perspective. Int J Eat Disord. 2003;33:143-54.

53. Rossotto E, Rorty-Greenfield M, Yager J. What causes and maintains bulimia nervosa? Recovered and nonrecovered women's reflections on the disorder. Eat Disord: J Treat Prev. 1996:4:115-27.

54. Nilsson K, Abrahamsson E, Torbiornsson A, Hägglöf B. Causes of adolescent onset anorexia nervosa: patient perspectives. Eat Disord. 2007:15:125-33.

55. Lacey J, Coker S, Birtchnell SA. Bulimia: factors associated with its etiology and maintenance. Int J Eat Disord. 1986:5:475-87.

56. Patton MQ. Qualitative research and evaluation methods. Thousand Oaks, CA: Sage Publications; 2002

57. Gergen K. An invitation to social construction. London: Sage Publications Ltd; 2009 .

58. White M, Epston D. Narrative means to therapeutic ends. New York: W.W. Norton; 1990.

59. Becker AE, Arrindell AH, Perloe A, Fay K, Striegel-Moore RH. A qualitative study of perceived social barriers to care for eating disorders: perspectives from ethnically diverse health care consumers. Int J Eat Disord. 2010;43:633-47.

60. Easter MM. "Not all my fault": genetics, stigma, and personal responsibility for women with eating disorders. Soc Sci Med. 2012;75:1408-16. 